\title{
ALGUNAS TAXONOMÍAS DEL LÉXICO DE LAS EMOCIONES Y SU PERTINENCIA PARA EL CORPUS LÉXICO DE UN ESTUDIO TRADUCTOLÓGICO INGLÉS-ESPAÑOL*
}

\author{
Jesús M. Sánchez García** \\ Universidad de Córdoba
}

\begin{abstract}
RESUMEN: Se exploran ciertas consideraciones y clasificaciones semánticas y cognitivas, relativas al léxico de las emociones, que han sido importantes para la estructuración de una dimensión del dominio del amor a fin de obtener un corpus léxico que resultara aplicable al estudio traductológico del tema del amor en la novela de Lawrence Durrell The Alexandria Quartet y en su traducción al español. En otras publicaciones ya se ha dado cuenta del marco general de este estudio y de los detalles de dicha estructuración, la cual sigue criterios procedentes del modelo lexemático-funcional de Leocadio Martín Mingorance y su Proyecto Lexicográfico. Asimismo, se resalta la importancia de que el traductológo (como todo lexicólogo que opere onomasiológicamente) se remita a enfoques léxico-semánticos y semántico-cognitivos, con objeto de estructurar con fiabilidad la dimensión que le sea útil para su interés aplicado.
\end{abstract}

ABSTRACT: Certain semantic/cognitive considerations and taxonomies are explored which are relevant to the lexis of emotion and which have played an important part in structuring one dimension in the domain of love to obtain a lexical corpus applicable to the descriptive study of the theme/topic of love in Lawrence Durrell's novel The Alexandria Quartet and its Spanish translation. The general framework for this research, as well as the details of such structuring, has already been dealt with elsewhere on the basis of criteria established in Dr. Leocadio Martín Mingorance's lexematic-functional model and in his lexicographic project. In addition, emphasis is laid on the translation scholar (or, for that matter, any lexicologist working along onomasiological lines) having recourse to lexico-semantic and semantico-cognitive approaches, if the domain dimension useful for his or her applied purpose is to be structured in a sound manner.

\section{Presentación}

El propósito de este artículo es tratar una serie de consideraciones y clasificaciones, relativas a los términos de las emociones, que han sido importantes en nuestra estructuración de lo que brevemente podemos llamar el campo o dominio del amor

* Este trabajo forma parte del proyecto de investigación "Desarrollo de una lógica léxica para la traducción asistida por ordenador a partir de una base de datos léxica inglés-francés-alemán-español multifuncional y reutilizable” financiado por la DGICYT, código PB94/0437.

** Doctor en Filología Inglesa, Depto. de Filologías Francesa e Inglesa. 
para obtener un corpus léxico que nos resultara aplicable al estudio traductológico textual del tema del amor en la novela de L. Durrell The Alexandria Quartet y en su traducción al español. En otras publicaciones ya he dado cuenta de esta investigación (Sánchez 1994, 1995a, 1995b y 1995c) y de los detalles de dicha estructuración (Sánchez 1995b) ${ }^{1}$ atendiendo a criterios procedentes del modelo lexemático-funcional de Leocadio Martín Mingorance (M. Mingorance 1990) y de sus aplicaciones lexicográficas. El artículo presenta aspectos concretos de dicha investigación en particular, ${ }^{2}$ pero creemos que puede servir de manera general para entender mejor la importancia de la semántica cognitiva para la semántica léxica si se parte de ésta para realizar un estudio traductológico textual de un tema narrativo cualquiera. De hecho, somos partidarios de un enfoque léxico-conceptual afin a las propuestas de Faber y Mairal (1995), trabajo en el que se propone una tipología de esquemas cognitivos afianzados en el significado léxico y en la arquitectura global del lexicón de una lengua natural tal y como los considera el modelo lexemático-funcional.

Dada la longitud del corpus textual, unas dos mil páginas, una preocupación fundamental era reducir el corpus léxico que nos diera la entrada informática en las novelas de forma que el resultado, la descripción traductológica, fuera lo más condensado posible, asentándose en la obtención de un corpus máximamente representativo de pasajes textuales que hemos llamado transémico (Sánchez 1995a) por utilizar el transema como unidad de descripción. ${ }^{3}$ En lo que sigue se describe fundamentalmente el proceso seguido para llegar a la estructuración definitiva del dominio a través de diversas consideraciones relacionadas con las taxonomías cognitivas y léxicas comentadas; finalmente se abordan las decisiones heurísticas posteriores que se tuvieron en cuenta para obtener el corpus léxico definitivo que se insertaría informáticamente en los dos textos de la novela, al objeto de delimitar el corpus transémico sobre el que realizar la investigación traductológica propiamente dicha. ${ }^{4}$

\footnotetext{
1. Sirvan las referencias señaladas como contexto general para todas las cuestiones concretas que se tratan en este artículo.

2. Uno de los objetivos era desarrollar una metodología de corte integrador y funcional, fundamentada en la teoría léxico-semántica y textual de forma multidimensional, y destinada a la descripción traductológica temática de textos narrativos. La metodología se aplicó a las relaciones traductivas léxico-semánticas micro y macroestructurales entre The Alexandria Quartet y su traducción al español (El Cuarteto para las dos versiones a partir de ahora), estudio que funciona en nuestra investigación global como piedra de toque y motor impulsor de la metodología. Para un recorrido por los presupuestos teóricos de dicha metodología y por el marco conceptual general en los que tienen cabida, veánse Sánchez (1996 y 1997).

3. Agrupamiento de pasajes textuales con textemas (Toury 1980) o unidades textuales identificadas según la relación traductiva (de semejanza o contraste) establecida cognitivamente entre ellas. En cuanto a la representatividad, utilizamos este concepto -fundamental en la delimitación y agrupamiento de los pasajes- en el mismo sentido que diversas teorías sobre la representación cognitiva del discurso narrativo (cfr. p.e. Kintsch 1977 y Meyer 1975) y creemos que los resultados metodológicos a los que ha dado lugar el interés por llegar a ella es una de las mayores aportaciones de nuestra investigación, junto con otras que tienen que ver con aspectos técnicos de la comparación intertextual, al anclar ésta en aspectos léxicos y conceptuales del modelo mencionado.

4. En realidad, la sola inserción en un conjunto de datos tan extenso como el mencionado, de tres lemas conceptuales (títulos léxicos entendidos tanto semémica como cognitivamente con independencia de la categoría sintáctica), ya produce problemas de delicada solución (véase el apartado final, donde se comenta ésta).
} 


\section{Inicios}

El estudio previo a la obtención del corpus léxico para un estudio traductológico posterior debe adecuarse al interés inicial que rige la selección del tema narrativo y por tanto del dominio léxico-conceptual correspondiente. En nuestro caso, los aspectos del amor que nos interesaba tratar hacen referencia a las relaciones emotivas ( $c f r$. infra) entre personajes en virtud de filias más o menos íntimas ( $c f r$. infra). Dentro del grupo de investigación de M. Mingorance, que se ocupa de la compilación de un lexicón onomasiológico contrastivo basándose en su modelo lexemático-funcional, P. Faber delineó tres dimensiones iniciales de los verbos de sentimiento que encajan perfectamente con el tipo de unidades léxicas cuyo estudio podría arrojar resultados elocuentes sobre el tema del amor, según lo percibido en unas primeras lecturas de la novela (en este caso El Cuarteto); es decir, es un tipo de trabajo que, junto con dicha intuición o percepción inicial, puede constituir un buen punto de partida cuando la investigación no es más que un esbozo desiderativo. Las dimensiones son:
a) dimensión de las sensaciones y emociones en general:
to feel. sentir.
b) dimensión del placer:
to enjoy. -disfrutar.
c) dimensión del amor y sus ingredientes eróticos:
to love; to desire.— querer, amar, enamorarse; desear.

\section{[TABLA 1: Esbozo inicial de dimensiones para el estudio traductológico del tema del amor]}

En principio, la primera categoría nos delimita el eje general del estudio; la segunda indica la escala o el eje axiológico de positividad en torno al que giran todos los demás lexemas de la dimensión que nos interesa en el dominio (es decir, prescindimos del eje negativo); y la tercera centra ya el análisis en las relaciones de amor y con el amor que mantienen los personajes en la novela.

\section{Taxonomías}

Trataremos primero las taxonomías que se adscriben a un paradigma cognitivo o que emplean una metodologa más cognitiva que puramente léxica, y posteriormente estas últimas.

\subsection{A. Ortony et al.}

Los autores investigan la elaboración de una extensa taxonomía léxico-conceptual de los estados afectivos (en especial de las emociones, que son tratadas como integrantes 
de aquéllos) sobre una base no empírica y partiendo de que se puede alcanzar una clasificación representativa mediante el análisis en componentes. Como otros estudios, el suyo (Ortony et al. 1987) considera diversas categorías o formas sintácticas (sobre todo sustantivos, adjetivos y verbos), las cuales no representarían diferencias semánticas apreciables sino que vendrían a ser manifestaciones del mismo concepto o "condición subyacente" (Ortony et al. 1987:356). Esta idea, acorde con nuestro propósito léxico-conceptual, está en consonancia con Aitchison (1987:99), quien no trata más que estas tres formas, pues entre ellas constituyen el léxico fundamental del vocabulario no gramatical.

En la propuesta de Ortony et. al. se señalan como los mejores ejemplares ${ }^{5}$ de la categoría de las emociones, es decir, como términos prototípicos, los que denotan condiciones mentales internas, son estados al mismo tiempo y su foco referencial es affect (por ej. happy) y no conducta ${ }^{6}$ o cognición (por ej. cheerful o encouraged). Sin embargo, aunque todas las emociones serían entonces afectos, no todos los afectos deben entenderse como emociones, de modo que affect podría ser un hiperónimo de emotion (vide infra para una discusión de la relación entre un término y otro).

Por su parte, muchos de los términos que denotan condiciones externas, aun cuando no se refieren a estados internos, sí poseen un fuerte elemento afectivo -sin llegar a ser la parte más significativa de su foco referencial-, ${ }^{7}$ como ocurre con sexy o lovable (evaluación subjetiva) y beloved (descripción objetiva). Pese a que éstos puedan parecer entonces más periféricos que happy, pleasure, o love, podría ser interesante su selección para un corpus léxico como el descrito más arriba, ya que son unidades que los autores consideran como emociones, aunque se encuentren ligeramente fuera de los focos predominantes que les resultan en general más interesantes (Ortony et al. 1987:353): afecto (happy, pleasure, in love, love), afecto-conducta (affectionate, loving, emotional, passionate) y afecto-cognición (desire), siendo la diferencia entre estos focos más de grado que de otra cosa. Agreguemos que según Lehrer (1990:374), si A es periférico con relación a $\mathrm{B}$ y éste lo es a su vez respecto de $\mathrm{C}$, puede que no se dé una relación transitiva entre las implicaciones lógicas de A, B y C en una taxonomía jerarquizada donde los lexemas hiperónimos mostrarían mayores efectos prototípicos que los hipónimos. Quizá sea éste el motivo de que percibamos el marco o esquema asignable a sex como menos relacionado con las emociones en general y más con la subdimensión emotivo-conductual erótica (positiva).

\subsection{J.A. Lee}

Su clasificación (Lee 1977) parte de trabajos experimentales, pero trata más los aspectos sociológicos que psicológicos de la experiencia del amor (entendido éste

5. Cfr. Rosch (1973) donde se delimita este concepto.

6. Véase más adelante la selección de dimensiones y lexemas que hacemos de la clasificación de Felices (1991) teniendo este criterio en cuenta.

7. Ya que tal vez dicho elemento podría describirse más correctamente como afectivo-causativo. 
como filia adulta íntima) para presentar una tipología estructurada de los "estilos" de amor en la que destacan seis conceptualizaciones dominantes: (1) EROS: búsqueda física de la imagen ideal de belleza. (2) LUDUS: permisividad, pluralidad, ausencia de celos. (3) ESTORGE: desarrollo del afecto y compañerismo, rechazo de la pasión autoconsciente, expectativa de compromiso a largo plazo. (4) MANIA: (eros + ludus): obsesión, celos, intensidad, repetición. (5) AGAPE: (eros + estorgé): altruismo, amor como deber personal sin expectativas de reciprocidad, cariño. (6) PRAGMA; (ludus + estorgé): consideración de los indicadores estadísticos de la pareja potencial.

\subsection{El estudio interlinguiístico de Tzeng et al.}

Tzeng et al. (1987) es un estudio de la estructura interna de las emociones donde se evalúan diez componentes de las denotaciones de emoción, así como su clasificación jerárquica con relación a su presencia en veintidós conceptos de emociones de veintitrés comunidades lingüísticas diferentes. Los componentes postulados determinarían la emoción en cuestión tanto para cada cultura como para la totalidad (Tzeng et al. 1987:443).

Los componentes son: Pleasantness (love, happiness, pleasure), Activation (passion), Control, Ego-orientation, Cognitivity, Overtness (passion), Dominance, Timidity, Terminality, Sociality (love).

\subsection{Johnson-Laird y Oatley}

En Johnson-Laird y Oatley (1989) se parte de una teoría cognitivo-comunicativa de las emociones sobre pruebas empíricas y reflejada en el léxico de las emociones, cuyo corpus contiene 590 términos; este léxico se estructuraría en un dominio semántico de forma relativamente simple si no fuera por la enorme diversidad de términos en los que se da un componente emocional. Asimismo examinan las implicaciones de dicha teoría para la semántica del léxico correspondiente, cuyo análisis es facilitado por la teoría, la cual encuadra a las emociones dentro de la mente -en relación con el yo- y dentro del grupo social -en relación con los demás-. Cualquiera de estas palabras es analizable y se basa en uno de los cinco "modos" de emociones subyacentes al léxico que postulan los autores y que vienen representados por títulos léxicos como por ej. happiness, además de otros cuatro (el único con valor positivo es happiness). Sólo uno de estos modos básicos no tendría estructura interna desde el punto de vista de su procesamiento mental.

La estructura del dominio semántico propuesto incluye siete categorías que recogen términos relativos a las emociones, dando siete tipos:

1) Genérico: emoción, pasión; denota emociones en general, cualquier emoción. Como el segundo modo, no precisa de una causa cognitiva discernible.

2) Términos emotivos básicos: denotan sentimientos experimentados sin conocer su causa u objeto (HAPPINESS). También incluye términos que denotan modificación de la intensidad (elation) y que pueden dejarse de lado por ser más periféricos. 
3) palabras que denotan relaciones emotivas: como suele ocurrir, la palabra alude al objeto o al origen de la emoción (experimentada en relación a otros individuos), como love; depende de la combinación entre el modo felicidad y el conocimiento de la persona hacia la que se siente la emoción.

4) emociones causadas: las palabras denotan emociones con causa conocida; por ej. pleasure.

5) causativos: concierne a la causa de la emoción; por ej. delight.

6) objetivos emocionales: concierne a algo que se tiene como objetivo, como desire. El logro del objetivo produce felicidad o placer.

7) emociones complejas: combinación de un modo emocional y una evaluación proposicional sobre algún aspecto del yo, esto es, hay conciencia de las circunstancias que producen las emociones, por ej. complacence, intimacy.

De estos siete tipos, tres parecen los más aplicables a un estudio temático como el ya aludido (hay que dejar a un lado, por tanto, el criterio de causa y las denotaciones genéricas). Son: relaciones emotivas, objetivos emocionales y emoción causada (coincidente con el resultado básico del logro de un objetivo).

\subsection{Storm y Storm}

En Storm y Storm (1987) se elabora una clasificación semántica del dominio de las emociones con base cognitivo-empírica en la que se hallan dos ejes dimensionales que afectan a las unidades de todo el dominio, marcando las relaciones que mantienen: placer-displacer (eje hedónico presente en todo estudio de emociones) y estímulosopor (Storm y Storm 1987:805-6). Mediante sucesivos análisis utilizando el método del agrupamiento jerárquico, Storm y Storm estudian la representatividad de un total de 577 términos relativos a las emociones (así como la semejanza relativa dentro de los grupos). La inclusión en categorías es aplicable no sólo a los propios términos sino también a los conceptos relacionados (Storm y Storm 1987:809), afirmación muy en la línea de la teoría de los marcos de Fillmore (por ej. 1977). Tras un primer agrupamiento resultaron dieciocho grupos o categorías iniciales, de los que podemos entresacar los siguientes para nuestro propósitos:

$\begin{array}{cl}\text { [Group] } & \text { [Term] } \\ 11 & \text { Affection } \\ & \text { Love } \\ & \text { Tenderness } \\ 13 & \text { Desire } \\ & \text { Lust } \\ 16 & \text { Pleasure } \\ & \text { Happiness }\end{array}$

Estos términos son los más generales e incluyen muchos otros, ya que los generales pueden reemplazarse por los más específicos con sólo una leve pérdida de significado. 
Los específicos tienen más rasgos analizables; para que un término ocupe un nodo superordinado las palabras subordinadas deben tener todos los rasgos semánticos de aquél, además de otros adicionales. Por ej.: sadness puede sustituirse por melancholy, disappointment, o grief. Por otra parte se admite en psicología evolutiva y cognitiva, y en psicología de la adquisición de lenguas (Storm y Storm 1987:814), que existe un nivel conceptual básico que se adquiere junto con el nivel léxico correspondiente antes que los niveles superiores o inferiores ${ }^{8}$, en los que interviene un proceso de diferenciación.

Los autores (Storm y Storm 1987:810) hacen la distinción entre referencia interpersonal (por ej., love) y no interpersonal (por ej., happiness), que afecta a los términos positivos. Estos a su vez representan una parte de la estructura general de la taxonomía. Términos positivos con referencia interpersonal serían:

- Love

- Lust, Desire, Horny, Passion, Sensual, Sexy, Seductive (+elemento sexual explícito)

- Attraction, Ardent, Infatuation, Romantic, Sentimental (+atracción intensa, -el. sexual explícito)

- Adoration, Devotion, Reverence, Worship (+subordinación ante el objeto de la emoción)

- Liking (puede o no entrañar amor)

- Admiration

- Sensitive, Warm (describen rasgos interpersonales o conducta interpersonal en general más que sentimientos)

- Affection, Tenderness (entraña protección)

- Pity, Compassion, Empathy, Sorry for, Sympathy. (entraña protección ante el sufrimiento del objeto)

\section{[TABLA 2: Términos positivos con referencia interpersonal según Storm y Storm]}

Love resultó primero en cuanto a prototipicidad según este y otros estudios mencionados por los autores.

Happy incluye para los autores otros términos más específicos como los del grupo que expresa reacciones a un suceso externo y continuo: Delight, Enjoy, Erotic, Pleasure, Sensuous.

Por tanto pleasure y eroticism estarían dentro de happiness. A nuestro entender los sujetos incluyen erotic en el experimento porque lo que se les presenta es un conjunto de palabras en vez de acepciones o predicados semánticos. De esta forma, erotic viene estrechamente asociado con pleasure sólo en un nivel concreto.

8. Nótese que básico no quiere decir aquí, como tampoco en Johnson-Laird y Oatley (1989), ni el más general ni el más particular (cfr. la discusión de básico en Lyons 1981, y Lakoff 1980 y 1987). 


\subsection{Koevecses}

Koevecses (1986) establece modelos populares del amor (folk models of love) con base lingüística, en los que menciona una serie de términos no ordenados que él estructura con criterios cognitivos. Su enfoque pretende revelar a través del léxico la porción concreta del sistema conceptual relativa al amor. Para ello considera importante tratar la metonimia, la metáfora y otros conceptos relacionados. Se basa en el concepto de Lakoff y Johnson (1980) de metáfora conceptual: el amor es una metáfora que se infiere mediante otras metáforas a partir de diversas expresiones. Cada metáfora refleja un aspecto del amor en términos de las correspondencias ontológicas entre las entidades de los dos dominios de la metáfora en juego y de las epistémicas entre nuestro conocimiento acerca del dominio origen típico y el que tenemos del dominio de llegada, el cual se obtiene a partir del dominio origen.

Koevecses analiza un corpus de metáforas para llegar a una definición clara del amor que opere dentro de los modelos cognitivos prototípicos típico e ideal. Al modelo ideal llega a partir del análisis de los sucesos, estados y propiedades relacionados con las metáforas (por ejemplo la metáfora central love is a unity), metonimias conceptuales (physical closeness indicates love) e implicaciones metafóricas del amor (consecuencias derivadas de las metáforas que extrae interpretativamente). El amor es conceptualizado entonces mediante diversas metáforas conceptuales que revelan otras emociones concomitantes, como el deseo sexual -al que el amor presupone metonímicamente-, el afecto o la ternura. Según esta visión, el concepto denotado por happiness sería un concepto inherente producto de la experiencia amorosa, que debería figurar en la definición de love junto con otros inherentes (enthusiasm, interest, longing, intimacy); como no es posible, debido al carácter convencional de las definiciones, el modelo cognitivo es más apropiado y da una visión más enriquecida que la definición, y tanto los conceptos asociados como los inherentes se incluyen en el modelo ideal. Así como las metáforas relativas al calor dan idea de la alta escala de intensidad que define al amor, otro grupo de metáforas nos habla de la relación entre el amor y el placer. Este es el modelo ideal que se deduce:

"1. True love comes along.

The other attracts me irresistibly.

The attraction reaches the limit point on the intensity scale at once

2.The intensity of the attraction goes beyond the limit point.

3.I am in a state of lack of control.

Love's intensity is maximal.

I feel that my love gives me extra energy.

I view myself and the other as forming a unity.

I experience the relationship as a state of perfect harmony.

I see love as something that guarantees the stability of the relationship.

I believe that love is a need

that this love is my true love.

that the object of love is irreplaceable. 
that love lasts forever.

Love is mutual.

I experience certain physiological effects: increase in body heat, increase in heart rate, blushing and interference with accurate perception.

I exhibit certain behavioral reactions: physical closeness, intimate sexual behaviour, sex, loving visual behaviour.

I experience love as something pleasant.

I define my attitude to the oject of love through a number of emotions and emotional attitudes: Liking, sexual desire, respect, devotion, self-sacrifice, enthusiasm, admiration, kindness, affection, care, attachment, intimacy, pride, longing, friendship, and interest.

I am happy." (Koevecses 1986:96)

\section{[TABLA 3: Modelo ideal del amor en Koevecses]}

\subsection{Wierzbicka}

Pasando ya a taxonomías más estrictamente léxicas, el interés de la taxonomía y definiciones de algunos términos de emociones que se propone en Wierzbicka (1972 y 1973) es que se utilizan criterios específicamente lexico-semánticos, aunque ya se menciona la necesidad de plantear el problema en términos más semántico-cognitivos y de describir las emociones mismas en primer lugar como clave de la estructura interna de los términos asociados. A diferencia de la estructura de los pensamientos, la de los sentimientos no puede describirse en palabras y lo único que se puede hacer es "describe in words the external situations or thoughts which are associated in our memory or in our imagination with the feeling in question and to trust that our reader or listener will grasp what particular feelings are meant" (Wierzbicka 1972:59).

La estructura léxico-semántica revela una descripción comparativa, pues sólo se identifica una emoción en función de la situación asociada ("sentirse como cuando...”), y correspondería a la semántica encontrar la descripción adecuada a tal comparación. En la única emoción interpersonal positiva que da (sympathy), en vez de describirse la emoción directamente, se da una definición en la que hay una identificación entre los sujetos de la experiencia. (Wierzbicka 1972:64).

La división entre emociones suaves (joy) y violentas (fear, anger) se basa en la distinción entre querer y desear. ${ }^{9}$ Happiness es tratado como un ideal humano universal, a diferencia de pleasure, y como representante de la serie seguida por joy, elation etc. Para la autora lo único que puede expresarse de una emoción es un correlato del sentimiento, es decir, el suceso que produce la emoción, el producido por ella o por reacciones fisio-

9. Por suavidad la autora entiende sutileza; es decir se está refiriendo a la cualidad con que se manifiesta esencialmente la emoción, y no a los efectos que las emociones pueden tener (violentos en ciertos casos de alegría o de deseo, como por ej. en "explosión de alegría” o "deseo arrebatador/bestial”). 
lógicas (Wierzbicka 1973:502). Como los estados de cosas causados por los sentimientos son lo bastante regulares, de forma que sirven para identificar a éstos, las definiciones que expresan los estados de cosas se deben proponer, a nuestro entender, como expresiones también relativamente estables. Así al analizar el uso de este dominio en Tolstoy, la autora nos indica que éste describe los efectos fisiológicos correlacionados con los sentimientos (expresión facial, sonrisa...) más que los sentimientos mismos. En realidad, creemos que lo que da la pista para que el lector infiera el concepto o significado literario de una emoción es la comprensión intersubjetiva, mediante imágenes cognitivas, del significado de las descripciones que se corresponden con los sucesos comunicados (los cuales a su vez han causado una emoción o son causados por ella).

Según todo lo expuesto, una consecuencia de las distintas taxonomías es que se puede justificar de momento la siguiente escala, la cual viene a perfeccionar notablemente el primer esbozo inicial (vide supra):

POSITIVE FEELING (pleasure-related happiness as criterial).
(SENTIMENT, EMOTION, SENSATION):
'PLEASURE-HAPPINESS'
LOVE
DESIRE
PASSION
SEX/SEXUALITY (EROTIC-ISM)
\{as elements of love
SENSUALITY
AFFECTION
TENDERNESS
\{as additional qualities or emotions

[TABLA 4: escala provisional de dimensiones procedente de las taxonomías] ${ }^{10}$

\subsection{La clasificación de A. Felices atendiendo al clasema axiológico.}

Como consideramos que los conceptos lexicalizados de las emociones están atravesados por la polaridad axiológica, definida por Krzeszowski (1990) en función del factor humano asociado a una lexicalización dada, nos parece oportuno tener muy en cuenta para nuestra estructuración del léxico de las emociones los criterios metodológicos de la clasificación de Felices (1991), que se realiza atendiendo al clasema axiológico, dentro del marco lexemático-funcional de M. Mingorance (por ej. 1990), y partiendo, como en nuestro caso, de las obras lexicográficas para extraer los datos sin clasificar. De hecho, encontramos una gran semejanza entre su línea de interés y la $1995 b)$.

10. Cfr. también nuestros comentarios sobre SENSATION, PLEASURE y HAPPINESS (Sánchez 
nuestra, especialmente en su tratamiento de los lexemas (aunque no todos ellos) que expresan valoración específica11, es decir, los de las emociones y los de la conducta.

A continuación indicamos tres dimensiones de las presentadas por Felices (1991) que guardan cierta relación con nuestro dominio a través de algunas de sus unidades integrantes y por tanto se podrían estudiar también en un análisis exhaustivo del tema del amor en cualquier novela y sus traducciones, aunque nosotros las tengamos que dejar por fuerza de lado, bien por no pertenecer a una superdimensión con el componente emotivo como componente central, bien por tratarse de una dimensión sólo periféricamente relacionada con la dimensión central desde la perspectiva de las emociones. Nótese, sin embargo, que los lexemas siguientes podrían en principio resultar interesantes de estudiar en virtud de la presencia de componentes como [_affection] o [_ sex].

1) SUPERDIMENSION: CONDUCTUAL ESPECIFICA

(_ attitude/behaviour); DIMENSION: ACTITUD ANTE LOS ASUNTOS SEXUALES:

Lexemas: obscene $[\in$ attitude/behaviour], $[\in$ toward sexual matters], $[\in$ negative],

$[\in$ offensively]

promiscuous $[\in$ attitude/behaviour], $[\in$ toward sexual matters], $[\in$ negative $]$, [ $\in$ frequent relationships]

faithful[ $\in$ attitude/behaviour], $[\in$ toward sexual matters], [ $\in$ positive]

[ $\in$ legitimacy/inactivity]

2) SUPERDIMENSION: CONDUCTUAL ESPECIFICA ( $\in$ attitude/behaviour); DIMENSION DE LA BONDAD EN LA CONDUCTA:

Lexemas: warm[ $\in$ attitude/behaviour], $[\in$ goodness in behaviour (toward others) $],[\in$ kindness y friendliness $]$, $[\in$ specific $],[\in$ affection $]$

3) SUPERDIMENSION: EMOTIVO-CONDUCTUAL COMBINADA [ $\epsilon$ emotion/behaviour]. DIMENSION: SENTIMIENTO Y ACTITUD DE DEBER ANTE LOS DEMAS:

Lexemas: faithful[ $\in$ emotion/behaviour combined] $[\in$ duty to the others], [ $\in$ fidelity], [ $\in$ sexual relat.]

[TABLA 5: Dimensiones de interés colateral para la estructuración del dominio del amor (Felices).]

Tres son las superdimensiones de las que consta el corpus léxico de A. Felices: la superdimensión emotiva específica, la emotivo-conductual combinada y la conductual específica. En el apéndice se reproducen los gráficos que representan la estructuración de las dimensiones que nos interesan, con los lexemas inicialmente más importantes para nuestro estudio en subrayado: la dimensión del sentimiento hedónico (8.1.1:

11. en contraposición a los que expresan valoración genérica. 
placer, felicidad), la del afecto (8.2.3: afecto, amor), y la del deseo (8.2.6/10.4: deseo, pasión). Los códigos numéricos son los mismos que en Felices (1991) para una mejor referencia. De los gráficos hemos extraído (Sánchez 1995b) una serie de adjetivos correspondientes a los subgrupos que tienen que ver con los lemas conceptuales intuidos al comienzo de la investigación o proporcionados por el proyecto lexicográfico aludido (de forma que hemos seguido la línea [ $\epsilon$ affection] $-[\in$ love]: amorous, sentimental, tender, devoted... en vez de $[\in$ affection $]-[\in$ liking $]$ : fond, likeable, popular).

Según los criterios de representatividad mencionados y para adecuar estos resultados a nuestro objetivo, podemos obtener los siguientes lexemas y dimensiones:

1- superdimension emotiva especifica

- subdimensión [experiencing]

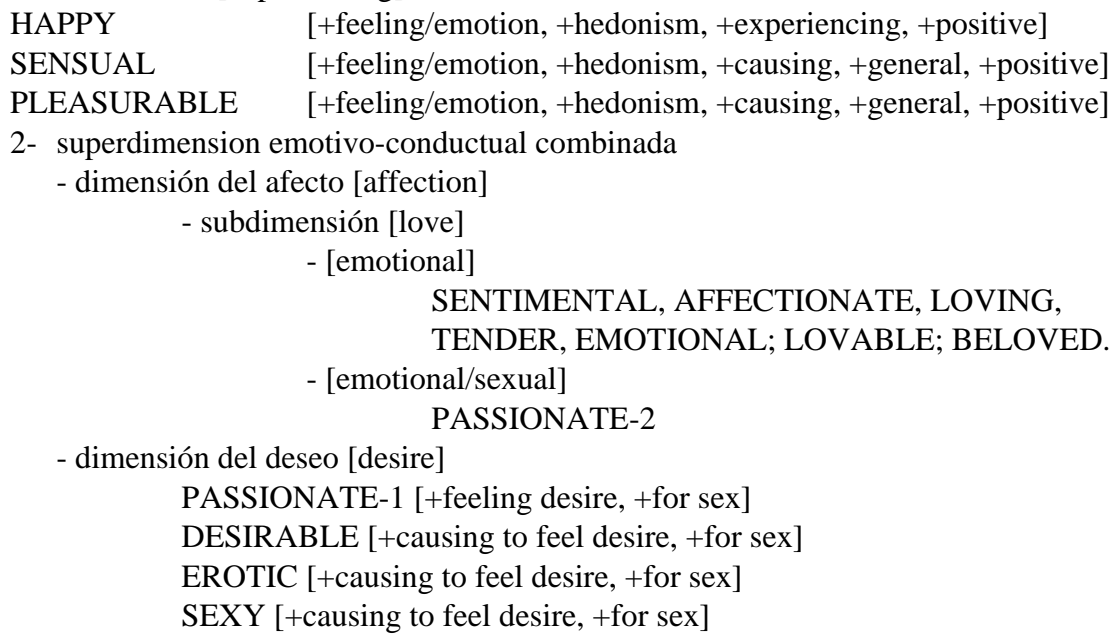

[TABLA 6: Dimensiones y lexemas procedentes de Felices (1991)]

\section{Conclusiones y consideraciones finales: Taxonomías y corpus léxico definitivo}

Este es el primer esbozo intuitivo de estructuración del que partimos, dado nuestro interés por el amor en el mundo de ficción de El Cuarteto como experiencia hedónica y, sobre todo, emotiva y sexual:

FEELING/SENSATION/EMOTION
PLEASURE (Positiveness)
LOVE
DESIRE (as libido)


SEX(UAL(ITY)(sexual interest, ie. the actual expression of libido) SENSUAL(ITY)

\section{[TABLA 7: Primer esbozo intuitivo de estructuración]}

Desde este planteamiento llegamos a la estructuración definitiva del dominio expuesta en Sánchez (1995b) (véase ejemplo en apéndice) incorporando a este esbozo -que se ve así modificado- los resultados que se desprenden de las consideraciones que ya hemos visto al tratar las taxonomías anteriores. Recapitulemos sus implicaciones para comprender los cambios introducidos en esta escala dimensional.

De la taxonomía de Ortony et al. hay que destacar que, puesto que las emociones son tratadas como integrantes de los estados afectivos, con affect como hiperónimo de emotion, la dimensión que perseguimos está nucleada en torno a los afectos que son también emociones, es decir en torno a las emociones afectivas. La aplicación de ambos conceptos o esquemas lexémicos (Faber y Mairal 1995) es muy coincidente y por tanto problemática. En el apéndice se ve como en este caso optamos por un criterio más estrictamente léxico no contraviniendo la tradición lexicográfica, que parece decantarse por entender affects no como un caso de meronimia en relación a emotion, sino de cuasi-sinonimia introduciendo un nuevo punto de vista.

De la tipología de Lee nos interesa la delimitación de nuestro tema como filia adulta íntima, la única que exploramos en nuestra investigación general del tema del amor en El Cuarteto y en su traducción al español. Es de destacar el interés psicológico de las categorías eros, estorgé y ágape, puesto de manifiesto en los modelos de Koevecses y en la representación del amor en la novela, que también trata -aunque más marginalmente- las variantes de ludus, manía, y pragma. Las tres primeras deben aparecer en la definición sistémica de los miembros del dominio del amor (vide apéndice), aunque sea veladamente en forma de la escala

\section{atracción (física o romántica) — intensidad — profundidad}

correspondiente a la polaridad

\section{identificación/atracción - desarrollo/transcendencia,}

propia de la conceptualización del amor en la cultura occidental ${ }^{12}$

El estudio de Tzeng pone de relieve la independencia mutua de los ejes afectivo y cognitivo en relación con las emociones y confirma la hipótesis de que hay correspondencia conceptual entre la arquitectura léxico-semántica de los dominios inglés y español elegidos. Ninguno de los componentes es totalmente irrelevante para las diferentes culturas y la importancia relativa entre los componentes es interlingüísticamente estable; por ello poseen un alto grado de validez en la representación semántica (Tzeng et al. 1987:460) y hacen viable la comparación. Asimismo se pone de manifiesto que los términos que nos interesan comparten todos una dimensión positiva y

12. Y que en el caso de Durrell, se retoma añadiendo tintes orientales. 
que la conceptualización del amor se refiere ante todo a una emoción de carácter social, es decir, interpersonal; estos dos extremos vienen a validar y confirmar nuestro interés por los correspondientes aspectos del amor como tema narrativo intuidos en nuestro planteamiento inicial.

De los siete tipos pertenecientes a la taxonomía de Johnson-Laird y Oatley, nos parece que hay que incluir sólo los más significativos: relaciones emotivas (love), objetivos (desire) y resultado básico del logro de un objetivo (happiness). A diferencia de los autores, nosotros sí remitimos happiness a su hiperónimo pleasure, que nos parece de los dos el más básico (Sánchez 1995b). ${ }^{13}$ En el estudio de estos autores la evaluación cognitiva de la causa constituye criterio, mientras que para nuestro estudio aplicado no es una consideración necesaria. Además, en cuanto a la noción de "básico" preferimos el planteamiento de Verschueren (1981:335), para el que cognitivamente básicos son manzana o guitarra; los demás son superordinados o subordinados. Pensamos que lo mismo puede decirse de love (lo que nos confirma Storm y Storm), es decir, términos como affection o eroticism podrían considerarse subordinados de love y pleasure como su superordinado. La taxonomía de estos autores ha influido por ejemplo en Fellbaum, quien clasifica (Fellbaum 1990) los verbos de las emociones (entre ellos love) siguiendo el análisis de sustantivos correspondientes a los cinco modos básicos de emociones y basándose en las funciones semánticas Experimentador y Origen de la emoción. Coincidiendo con nuestro enfoque, Fellbaum extrae también el estudio de una parte de la oración del de la clasificación previa de otra distinta, en este caso la de los sustantivos. En nuestro estudio consideramos los sustantivos como títulos léxicos representantes de los conceptos que denotan pero partimos en gran medida del estudio del adjetivo para desarrollar después las demás clases.

La distinción interpersonal/no interpersonal que afecta a los términos positivos de Storm y Storm es sumamente importante y determina ciertas decisiones prácticas a

13. Esta circunstancia puede observarse en la definición de cada lexema, que se propone según el método de la descomposición léxica gradual de S. Dik, asumido por el modelo lexemático-funcional. La discrepancia con las propuestas cognitivas en este punto llama la atención sobre un punto de importancia dentro de un enfoque léxico-conceptual: que ambos polos del enfoque deben vigilarse y corregirse mutuamente si se les quiere hacer justicia. Cabría preguntarse entonces sobre la causa de que suela aparecer happiness con una aplicación más general que pleasure en los resultados de los diversos trabajos experimentales. Es esta una cuestión que tambien atañe a la antropología cognitiva y cultural y que expone los riesgos de adscribirse excesivamente a los enfoques puramente cognitivos. En efecto, se podría pensar que los sujetos de la investigación, situados ante los diversos tests y preguntas, activan presupuestos más culturales que lingüísticos sobre el significado de los términos, anteponiendo happiness a pleasure en la escala de generalidad y especificando éste en términos de aquél en virtud de una postura idealista, predominante en nuestra cultura, que coloca la felicidad en lo alto de un pedestal y se muestra renuente a considerar el placer como positivo, considerándolo mucho menos pertinente en términos culturales y dejándolo por tanto en un lugar muy inferior. Sin embargo, si la felicidad es un ideal humano universal, no lo es menos la búsqueda del placer. 
la hora de confeccionar el corpus léxico (véase más abajo). De la lista de términos positivos con referencia interpersonal podríamos escoger los más representativos, dejando: love, desire, passion, sensual, sexy, sentimental, sensitive, affection, tenderness. Estos podrían clasificarse como productores de felicidad o como elementos de ella y por consiguiente como hipónimos de happiness/pleasure. Love y liking incluyen aquí a los demás pero también se podría entender que el primero es hipónimo del segundo, como hace Faber en su clasificación de los verbos de sentimiento (vide supra). De hecho, esta es la lista a la que llegan los autores después de la aplicación de una serie de criterios. En la fase inicial de identificación de los primeros grupos, se seleccionan 72 términos y los sujetos agrupan love, affection y tenderness, rompiendo con la distinción love vs. liking de manera que todos estos términos también podrían incluirse bajo love como conceptos que son formas del amor. El estudio confirma, pues, que el placer es un tipo de sentimiento/emoción/sensación como base asociativa de la felicidad, en el que podemos encontrar emociones placenteras como amor y sus conceptos hiponímicos relacionados (afecto, deseo, etc.). Sin embargo, las emociones eróticas (deseo, pasión...) pueden contenerse o no en love como conceptos metonímicos relacionados, dependiendo de si se trata de amor romántico o sexual, siendo quizá mejor estructurarlas como emociones independientes en el sistema, aunque en realidad suelan ser concomitantes a love.

Puesto que los modelos cognitivos de Koevecses vendrían a estar entre la fórmula del tesaurus y el dominio estrictamente semántico, teóricamente sería interesante elaborar un corpus léxico tan flexible como el modelo cognitivo, es decir, contar con el mayor número de términos incluidos en éste para efectuar posteriormente la comparación con el texto. Pero los modelos son extensos, por lo que hay que arbitrar otro criterio de selección para el corpus léxico que sin embargo debería ser conceptualmente compatible con los modelos cognitivos. En su estudio, la metáfora central es love is a unity. Creemos que debe darse cuenta de alguna forma de esta característica en la definición léxica, y que el componente "attraction" le hace justicia, ya que la unidad establecida se entiende como resultado de un movimiento mutuo hacia el otro, un coming together. En la tabla 3, en negrita, figuran conceptos muy pertinentes para nuestros propósitos; nuestra elección final es entonces léxico-conceptualmente compatible con el modelo ideal. Lógicamente puede considerarse que esta compatibilidad es parcial, lo que no debe desanimarnos pues es sabido que resulta inviable incorporar las propuestas cognitivas en toda su extensión y ámbito a los estudios lexicográficos o lexicológicos aplicados. Las implicaciones metafóricas las extrae el autor de forma interpretativa, lo que llama la atención sobre el carácter interpretativo-intersubjetivo de los esquemas, modelos o marcos experienciales del cognitivismo y de gran parte de la semántica léxica. El amor es conceptualizado en diversas metáforas conceptuales que revelan otras emociones concomitantes o metonímicas como el deseo sexual o el afecto y la ternura. El concepto denotado por happiness sería producto de la experiencia amorosa y debe figurar de algún modo en la definición de love. También se resalta 
el grado muy elevado en la escala de intensidad, así como la relación entre el amor y el placer. Semas y sememas extraíbles del modelo ideal (entre otros) son: attraction, intensity, unity, sex, pleasant, sexual desire, happy.

En cuanto a la taxonomía de Wierzbicka, el elemento de identificación entre sujeto y objeto se puede incorporar a las definiciones en forma de rasgos como "positividad", "atracción”, "relación sexual", "unión carnal”. La importante distinción entre emociones violentas y suaves (querer, desear) está presente en nuestro dominio entre love, affection, etc. y las emociones eróticas (vide apéndice). En cuanto al tratamiento de happiness, estamos de acuerdo en que se trata de un título (o conjunto de términos asociados) que representa a muchos otros por funcionar como valor por defecto. Sin embargo, como ya se anticipó, discrepamos con esta autora sobre la relación entre este término y pleasure.

Por su parte, la obra de Felices aporta a nuestra propuesta un sistema riguroso para llegar a las siguientes dimensiones conceptuales, ${ }^{14}$ las cuales subyacen a los correspondientes elementos léxicos de dicha estructuración:

1- HAPPINESS/PLEASURE/SENSUALITY

[emoción específica +hedonismo +eudemonismo general]

2-

SENTIMENT [ $\rightarrow$ FEELING], EMOTION, LOVE,

AFFECTION, TENDERNESS, PASSION-2

[emoción-conducta combinadas +afecto +amor +emoción

_el. sexual (gen) ${ }^{15}$ ]

3-

\author{
EROTICISM \\ PASSION-1 \\ DESIRE \\ SEXUALITY/SEXINESS \\ [emoción-conducta combinadas +deseo +el. sexual]
}

\title{
[TABLA 8: Dimensiones conceptuales finales]
}

El título del dominio de los adjetivos que nos interesa extraer de su análisis podría ser "adjetivos que expresan sentimiento positivo o emoción positiva produciéndose algún tipo de placer o felicidad”. De ahí que nuestro dominio, informalmente llamado del amor, sea más bien la dimensión de los sentimientos y emociones positivos. En apéndice incluimos también un ejemplo de cómo queda la estructuración definitiva (solamente sustantivos en inglés) ${ }^{16}$.

14. expresadas en sustantivos derivados de los adjetivos expuestos. Cfr. Sánchez (1995b) para la justificación de este procedimiento.

15. Excepto para love; veáse su definición y la de sus derivados en el apéndice y en Sánchez (1995b).

16. En ella se puede observar una enorme influencia del estudio cognitivo-taxonómico descrito en este trabajo, es decir, un alto índice de adecuación cognitiva, pero también ligeras discrepancias causadas por el empleo del modelo lexemático-funcional como irrenunciable criterio estrictamente lexicológico. 
Con objeto de ilustrar un procedimiento práctico para obtener un corpus léxico definitivo sobre el que realizar la investigación traductológica, nos centramos ya en lo que es nuestro estudio de las apariciones (lexical occurrences) en la rueda léxica de un programa de recuperación textual como WordCruncher, una vez que se cuenta con una versión electrónica de $E l$ Cuarteto en inglés y español.

De la lista total de unidades que pertenecen al dominio como queda estructurado (Sánchez 1995b), descartamos para el estudio traductológico las que presentan baja productividad en la rueda léxica de WordCruncher en cada idioma y en todas las clases tratadas (sustantivos, adjetivos y verbos), procediendo así con ambos idiomas independientemente.

En teoría no habría necesidad de descartar ninguno de los lexemas centrales de todas las clases, pero se hace así sólo por motivos prácticos, ya que según un propósito más general de la investigación, ésta no pretende ser más que ilustrativa de lo que sería un corpus transémico claro y máximamente representativo. En las tablas que siguen indicamos con asterisco los lexemas que hemos considerado definitivamente para su inserción en el programa, una vez que todas las clases léxicas se acumulan mediante su relación morfológica y en algún caso semántica (se incluye enjoy en PLEASURE y gozar y disfrutar en PLACER) formando lo que hemos denominado lemas conceptuales.

Esta es la lista obtenida a partir de las consideraciones anteriores. A la izquierda se da el lema conceptual y a la derecha los lexemas correspondientes con la categoría sintáctica a la que pertenecen.

\section{INGLES:}

HAPPINESS: $\quad$ happiness (s.); happy (adj.);

SENSUALITY: $\quad$ sensuality (s.); sensual (adj.);

EMOTION: $\quad$ emotion (s.); emotional (adj.);

LOVE: $\quad$ love, lover (s.); to love (vb.); lovable, loving, beloved (adj.);

FEELING: $\quad$ feeling, sentiment, sentimentalism (-ist) (s.); to feel (vb.); sentimental (adj.);

\footnotetext{
FEELING y EMOTION representarían dos subdimensiones obvias de la dimensión referida, aunque la relación entre estos dos títulos de subdimensión (conceptos y correspondientes términos) es problemática sea cual sea el criterio manejado, cognitivo o lexicológico, quizá debido a la propia polisemia de feeling (feeling como sensación -sensation vs. feeling como sentimiento -emotion-). Por ello hemos preferido clasificar EMOTION a la misma altura que FEELING y considerar así los hipocánones sensoemotivo y senso-erótico como casos independientes (FEELING=EMOTION), es decir, como ligeramente aislados del hipocanon más propiamente dependiente del FEELING en general, el senso-hedónico (FEELING=SENSATION).
} 
AFFECTION: $\quad$ affection (s.); affectionate (adj.);

TENDERNESS: $\quad$ tenderness (s.); tender (adj.);

DESIRE:

desire (s.); to desire (vb.); desirable (adj.);

PASSION: passion (s.); to impassion(ate) (vb.); passionate (adj.);

SEXUALITY:

EROTICISM:

sexuality, sex, sexiness (s.); sexual, sexy (adj.);

PLEASURE:

eroticism (s.); to eroticize (vb.); erotic (adj.);

pleasure (s.); to enjoy (vb.); pleasurable (adj.);

\section{ESPAÑNL:}

FELICIDAD: $\quad$ felicidad (s.); feliz (adj.);

SENSUALIDAD: $\quad$ sensualidad (s.); sensual (adj.);

EMOCION: $\quad$ emoción (s.); emocionarse (vb.);

emocional, emotivo (adj.);

AMOR: $\quad$ amor, amante (s.); amar, querer, enamorarse (vb.);

CARIÑO cariño (s.); encariñarse (vb.); cariñoso (adj.);

SENTIMIENTO: $\quad$ sentimiento (s.); sentir (vb.); sentimental (adj.);

AFECTO: $\quad$ afecto (s.); afectuoso (adj.);

TERNURA: $\quad$ ternura (s.); enternecerse (vb.); tierno (adj.);

DESEO: $\quad$ deseo (s.); desear (vb.); deseable (adj.);

PASION: $\quad$ pasión (s.); apasionarse (vb.); apasionado (adj.);

SEXUALIDAD: $\quad$ sexualidad, sexo (s.);

EROTISMO: $\quad$ erotismo (eros) (s.); erotizar (vb.); erótico (adj.);

PLACER: $\quad$ placer (s.); disfrutar, gozar; placentero (adj.);

[TABLA 9: Lemas conceptuales para la inserción procedentes del estudio anterior]

En la aplicación de estos lemas a El Cuarteto, los valores de las tablas que siguen corresponden a los ofrecidos inicialmente por el programa independientemente de las formas ambiguas, que se han de desambiguar durante la selección de referencias (por ej.: "siento" < sentir o sentar).

$\begin{array}{ll}\text { HAPPINESS } & 30,25,32,25=112 \\ \text { SENSUALITY } & 6,2,2,7=17 \\ \text { EMOTION } & 21,14,17,23=75 \\ \text { LOVE } & 251,229,159,140=779 \\ \text { FEELING } & 119,136,276,185=716 \\ \text { AFFECTION } & 11,14,27,18=70 \\ \text { TENDERNESS } & 28,20.22,17=87\end{array}$




$\begin{array}{ll}\text { DESIRE } & 40,28,29,27=124 \\ \text { PASSION } & 30,32,35,24=121 \\ \text { SEXUALITY } & 29,15,10,17=71 \\ \text { EROTICISM } & 0,0,0,2=2 \\ \text { PLEASURE } & 39,33,55,31=158\end{array}$

[TABLA 10: Valores iniciales para los lexemas ingleses por volúmenes del Cuarteto, independietemente de la selección de referencias]

$\begin{array}{ll}* \text { LOVE } & 195,179,112,115=* 605 \\ \text { *PASSION } & 22,27,26,14=* 89 \\ \text { *SEXUALITY } & 27,13,10,16=* 66 \\ \text { DESIRE } & 22,6,11,5=44 \\ \text { TENDERNESS } & 13,3,9,5=30\end{array}$

[TABLA 11: Tabla comparativa para la selección final. Valores por volúmenes del Cuarteto correspondientes a las referencias seleccionadas para el análisis transémico (inglés)]

$\begin{array}{ll}\text { FELICIDAD } & 33,22,19,43=117 \\ \text { SENSUALIDAD } & 8,3,3,6=20 \\ \text { EMOCION } & 22,18,17,29=86 \\ \text { AMOR } & 367,227,345,294=1233 \\ \text { CARIÑO } & 7,4,9,3=23 \\ \text { SENTIMIENTO } & 227,167,316,195=905 \\ \text { AFECTO } & 16,24,23,22=85 \\ \text { TERNURA } & 30,19,26,23=98 \\ \text { DESEO } & 70,53,46,40=209 \\ \text { PASION } & 26,33,35,22=116 \\ \text { SEXUALIDAD } & 55,15,10,17=97 \\ \text { EROTISMO } & 0,1,3,4=8 \\ \text { PLACER } & 27,23,31,30=111\end{array}$

[TABLA 12: Valores iniciales para los lexemas ingleses por volúmenes del Cuarteto, independietemente de la selección de referencias]

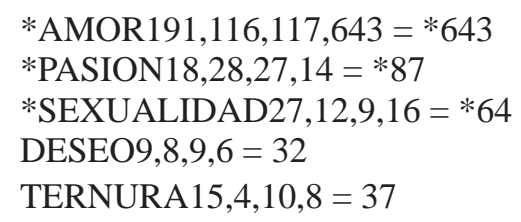

[TABLA 13: Tabla comparativa para la selección final. Valores por volúmenes del Cuarteto correspondientes a las referencias seleccionadas para el análisis transémico (español)] 
Las tablas 11 y 13 expresan las apariciones de cada unidad en la novela y pueden ser tomadas como pistas sobre por dónde discurre la novela. No obstante, hay una gran disparidad entre los valores debido especialmente al alto índice de polisemia de algunos de estos lemas. El trabajo para el analista debe simplificarse puesto que el programa sólo nos da palabras-ejemplar (tokens) y no palabras-tipo. Como la piedra angular es el amor como tema lingüístico-literario, y dadas ciertas consideraciones crítico-literarias nada opacas en el sentido de que la dimensión hedónica funciona en la novela como un sobreentendido o telón de fondo sobre el que los personajes dramatizan sus emociones, parece preferible centrarse en las dimensiones marcadas por los hipocánones senso-emotivo (amor como emoción) y senso-erótico (amor como filia íntima), y no tocar el hipocanon senso-hedónico, cuya exploración requeriría una inversión de tiempo y energía difícilmente disponible (téngase en cuenta el enorme corpus que supone el objeto de estudio para un sólo traductólogo); podemos así apartar de la lista de las tablas 11 y 13 los lemas PLEASURE, HAPPINESS y SENSUALITY (junto con los españoles). FEELING y EMOTION son de caracter archilexémico y genérico, mientras que AFFECTION Y EROTICISM son los que tienen una productividad más baja entre los restantes, aunque sería interesante también analizar AFFECTION por su proximidad a los demás. Lo mismo es aplicable al dominio español.

Todo ello, que nos da ya una idea del carácter eminentemente heurístico de este tipo de investigación aplicada, nos deja finalmente cinco lemas para el corpus léxico definitivo. Sin embargo hemos utilizado un nuevo criterio para reducir aún más nuestro ámbito de estudio, ya que separar por ejemplo SEXUALITY en favor de DESIRE y TENDERNESS en razón de su productividad relativa resulta contra toda intuición tras varias lecturas de la novela y teniendo en cuenta los estudios de la crítica especializada en el Cuarteto. Por tanto, hemos recurrido a un criterio más riguroso que el de los valores iniciales de WordCruncher: el de los valores obtenidos, una vez que se ha procedido a la selección de referencias ${ }^{17}$. Estos valores vienen expresados en las tablas 12 y 14. Ahora, PASSION y SEXUALITY resultan ser, con diferencia, los lemas conceptuales más interesantes de analizar entre los cinco mencionados, junto con LOVE, que, como es lógico, es apreciablemente el lema más socorrido para L. Durrell. Los tres son representantes de los dos parámetros de J.Laird y Oatley en los que decidimos centrarnos: emoción como relación y emoción dirigida a un objeto ${ }^{18}$.

Para finalizar, nos parece importante destacar que el traductológo que utilice una metodología léxica semejante a la nuestra, y en realidad todo lexicólogo que opere onomasiológicamente para estructurar con fiabilidad el dominio o dimensión que le

17. Procedimiento que se explicará en una futura publicación en otro artículo (de próxima aparición -1997- en un volumen dedicado in memoriam a L. M. Mingorance al cuidado de G. Wotjak, Leipzig) y que supone un inevitable acto interpretativo-crítico o act of scholarship.

18. Aún así se debería tener todo esto en cuenta al encontrar ejemplares pertenecientes a DESIRE y TENDERNESS en el análisis correspondiente de los pasajes textuales. 
sea útil para su interés aplicado, debe remitirse a estudios tanto léxico-semánticos como semántico-cognitivos ${ }^{19}$ o ejecutarlos él mismo sobre idéntica base. ${ }^{20} \mathrm{~A}$ la luz de lo expuesto ha quedado de manifiesto en el análisis de nuestro "dominio del amor", a través de las diferentes propuestas y esbozos, la proximidad o compatibilidad (ya que no total semejanza) revelada por los enfoques de ambas orientaciones. Asimismo, se ha indicado la forma en que éstos se pueden integrar, articulando sus aportaciones para llegar a una propuesta definitiva no contradictoria con la intuición inicial que da lugar al estudio traductológico y que siempre parte de la lectura de la novela, es decir, de la representación cognitiva de su tema principal (el amor en este caso). Creemos que la propuesta definitiva de estructuración del dominio, e inclusión de elementos léxicos, independientemente de las decisiones heurísticas ulteriores, sólo se puede considerar representativa, y por lo tanto manejable, si invocamos un criterio cognitivo que la remita a estudios y taxonomías como los comentados. Al mismo tiempo, la dispersión o diversificación de éstos debe ser regulada por criterios más puramente lingüísticos según un modelo lexicológico determinado.

\section{Bibliografía}

AITCHISON, J. (1987): Words in the Mind, an Introduction to the Mental Lexicon, Oxford: B. Blackwell, .

FABER P. y MAIRAL, R., (1995): "Semantic Frames and Dimensions. Towards a Unified Approach" International Journal of Lexicography, (en prensa).

FELICES LAGO, A. (1991): El componente axiológico en el lenguaje; su configuración en los adjetivos que expresan emociones y conducta en la lengua inglesa, Universidad de Granada, (tes. doct.).

FELLBAUM, C. (1990): "English Verbs as a Semantic Net" International Journal of Lexicography, (núm. esp.), 3 (4) pp. 278-301.

JOHNSON-LAIRD, P. N. y OATLEY, K. (1989): "The Language of Emotion: An Analysis of a Semantic Field", Cognition and Emotion, 3 pp. 81-123.

KINTSCH, W. (1977): “On Comprehending Stories”, en M.A. Just y P.A. Carpenter (eds.) Cognitive Processes in Comprehension, Hillsdale, N.J.: L. Erlbaum.

19. La escasez de este tipo de estudios en una de las dos lenguas relacionadas puede hacer que se dependa inevitablemente de los estudios realizados en una sola lengua, en nuestro caso el inglés. Este artículo presenta sus consideraciones desde el inglés, pero teóricamente habría que hacer lo mismo desde el español.

20. Emerge así el enfoque léxico-conceptual de base que reivindicamos tanto para el análisis en el sistema del significado léxico (de forma coincidente con Faber y Mairal 1995) y de las posibles equivalencias interlingüísticas existentes, como para una aproximación multifuncional e intersubjetiva a dichos significados en el texto y en su representación cognitiva. 
KOEVECSES, Z. (1986): Metaphors of Anger, Pride and Love: A Lexical Approach to the Structure of Concepts, Amsterdam: John Benjamins.

KRZESZOWSKI, T.P. (1990): "The Axiological Aspect of Idealized Cognitive Models", en J. Tomaszczyk y B. Lewandowska-Tomaszczyck (eds.), Meaning and Lexicography, Amsterdam: John Benjamins, pp. 135-165.

LAKOFF, G. y JOHNSON, M. (1980): Metaphors We Live By, Chicago: Chicago University Press.

LAKOFF, G. (1987): Women, Fire and Dangerous Things. What Categories Tell Us About The Nature of Thought, Chicago: University of Chicago Press.

LEE, J.A. (1977): “A Typology of Styles of Loving”, Journal of Personality and Social Psychology: Attitudes and Social Cognition Section, 3 pp. 173-182.

LEHRER, A. (1990): "Prototype theory and its implications for lexical analysis", en S.L. Tsohatzidis (ed.), Meanings and Prototypes. Studies in Linguistic Categorization. Routledge: Londres and New York, pp. 368-381.

LYONS, J. (1981): Language, Meaning and Context, London: Longman.

MINGORANCE, L. M. (1990): "Functional Grammar and Lexematics in Lexicography”, en J. Tomaszczyk y B. Lewandowska-Tomaszczyck (eds.), Meaning and Lexicography, Amsterdam: John Benjamins, pp. 227-253.

MINGORANCE, L. M. (1994): "Predicate Schemata and Cognitive schemata in Speech Production and Interpretation. Towards an Integration of ICM's in an FG Lexicon". Conferencia pronunciada en el VI Congreso Internacional de Gramática Funcional, York, agosto de 1994 (en prensa).

MEYER, B.J.F. (1975): The Organization of Prose and its Effects on Memory, Amsterdam: North Holland.

ORTONY ET AL., A. (1987): "The Referential Structure of the Affective Lexicon", Cognitive Science, 11(3) pp. 341-364.

ROSCH, E. (1973): "Natural Categories", Cognitive Psychology, 4 pp. 328-350.

SANCHEZ, J.M. (1994): Desplazamientos de traducción en El Cuarteto de Alejandría de Lawrence Durrell: un ejercicio en traductología descriptiva con un enfoque funcional combinado, Granada: Universidad de Granada, (tes. doct.).

SANCHEZ, J.M. (1995a): "El componente léxico y su función en el estudio traductológico textual”, en RESLA (Revista Española de Lingüística Aplicada), 10 (en prensa).

SANCHEZ, J. M. (1995b): “Una estructuración del campo léxico-conceptual del amor previa a su estudio traductológico inglés-español en The Alexandria Quartet", Atlantis (en prensa).

SANCHEZ, J. M. (1995c): "Desplazamientos léxico-semánticos y efectos macroestructurales en la traducción española de The Alexandria Quartet: topología conceptual", Miscelánea, 16 pp. 189-213.

SANCHEZ, J. M. (1996): "Fundamentos para una metodología descriptiva en el estudio traductológico del texto narrativo: I. Puntos de partida", Translatio (F.I.T.: París), XV (3-4). 
SANCHEZ, J. M. (1997): "Fundamentos para una metodología descriptiva en el estudio traductológico del texto narrativo: II. Condiciones de aplicación”, Translatio (F.I.T.: París), XVI (en prensa).

STORM, C. y STORM, T. (1987): “A Taxonomic Study of the Vocabulary of emotions", Journal of Personality and Social Psychology: Attitudes and Social Cognition Section, 53 (4) pp. 805-816.

TOURY, G. (1980): In Search of a Theory of Translation, Tel Aviv: Porter Institute.

TZENG ET AL., O.C.S. (1987): “Cross-Cultural Componential Analysis on Affect Attribution of Emotion Terms" Journal of Psycholinguistic Research, 16 (5) pp. 443-465.

VERSCHUEREN, J. (1981): "Problems of Lexical Semantics" Lingua, 53 pp. $317-$ 351.

WIERZBICKA, A. (1972): “Emotions”, en A. Wierzbicka Semantic Primitives, Frankfurt: Atheneum Verlag, pp. 57-70.

WIERZBICKA, A. (1973): "The Semantic Structure of Words for Emotions", en R. Jakobson, et. al. (eds.), Slavic Poetics: Essays in honor of Kiril Taranovsky, The Hague: Mouton, 1973, pp. 409-505. 


\section{APÉNDICES}

\section{Ejemplo de estructuración}

\section{Perception through the senses}

1.sensation: general perception through the senses (Archilex.)

1.1 feeling: sensation accompanied by a not primarily sensory response. (Archilex.)

1.1.1 sentiment: exaggerated or artificial feeling

1.1.2 pleasure: positive feeling when experiencing sth. that pleases the mind

1.1.2.1 happiness: deep pleasure that is intense or lasting

1.1.2.2 sensuality: pleasure esp. sexual experienced by the (strong gratification of) physical senses

1.2 emotion: strong feeling marked by excitement or agitation of the mind

1.2.1 affects: emotions esp. seen as states or functioning of the mind

1.2.2 love: positive deep emotion that is very intense and characterised by strong attraction (physical or romantic) towards sb.

1.2.3 passion 2: strong emotion of an intensity between affection and love.

1.2.4 affection: positive emotion of an intensity between sympathy and warmth felt towards other people

1.2.4.1 tenderness: affection that is gentle and caring

1.2.5 eroticism (Eros): (group of) instinctual emotions that tend to self-affirmation and uninhibited physical pleasure in physical love

1.2.5.1 passion 1: (set of) violent erotic emotions, strongly felt esp. in intense sexual love

1.2.5.2 desire: erotic emotion consisting in urgently wanting to have sex with sb.

1.2.5.3 sex: $\quad$ erotic emotions resulting from the urge to gratify the instinct of having physical intercourse with another organism.

1.2.5.3.1 sexuality: sex, esp. preoccupation, behaviour or potency associated with it. 
8.1. La superdimensión emotiva específica

8.1.1. La dimensión hedónica y su clasificación:

$[\in$ HEDONISM $]$

8.1.1.1. Subdimensión: [ $\in$ EXPERIENCING]

GRAFICO DE LA ESCALA SUBDIMENSIONAL POSITIVA:

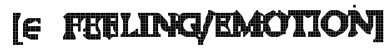

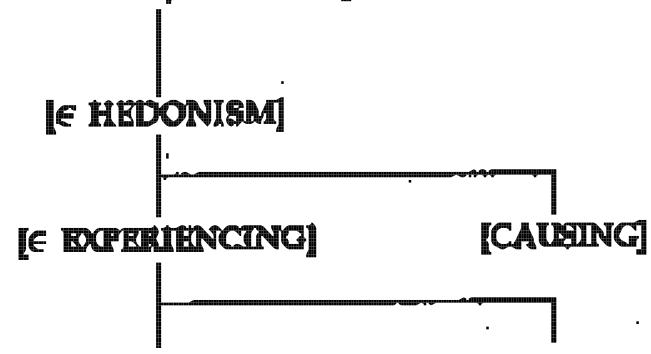

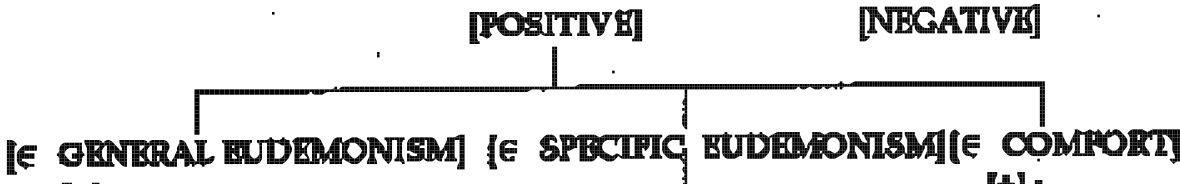

W!

Counlents

Conthented

Glud

Gathifed

H:

Rrupyy

Meniy

Flensed

Rellewed

Batlefined

$\llbracket \$$

enilted

jogfal.

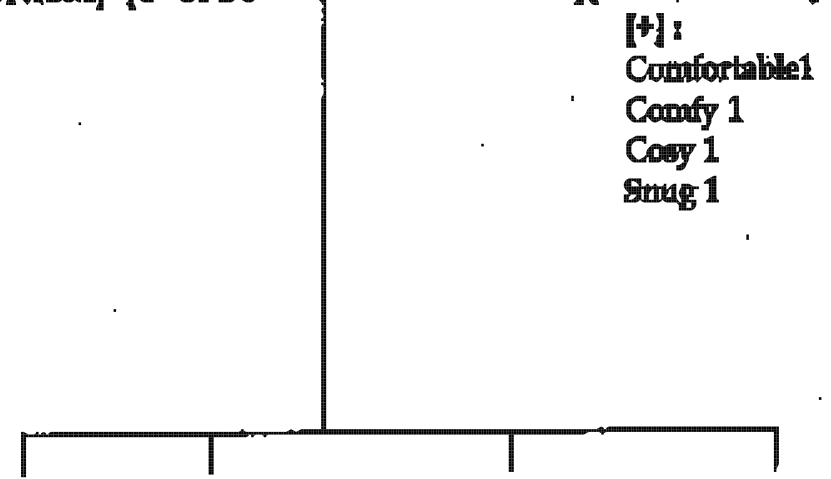

oweroped

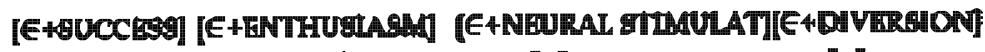

$1+1$

[+1:

[4]:

Bralbant

Bostatilie:

Delatied

[+]:

Jogoug 1

Elinted

Budied

$[++1$ :

Euphorle 
GRAFICO DE LA SUBDIMENSION HEDONISTICA

CAUSATIVA, ESCALA POSITIVA:

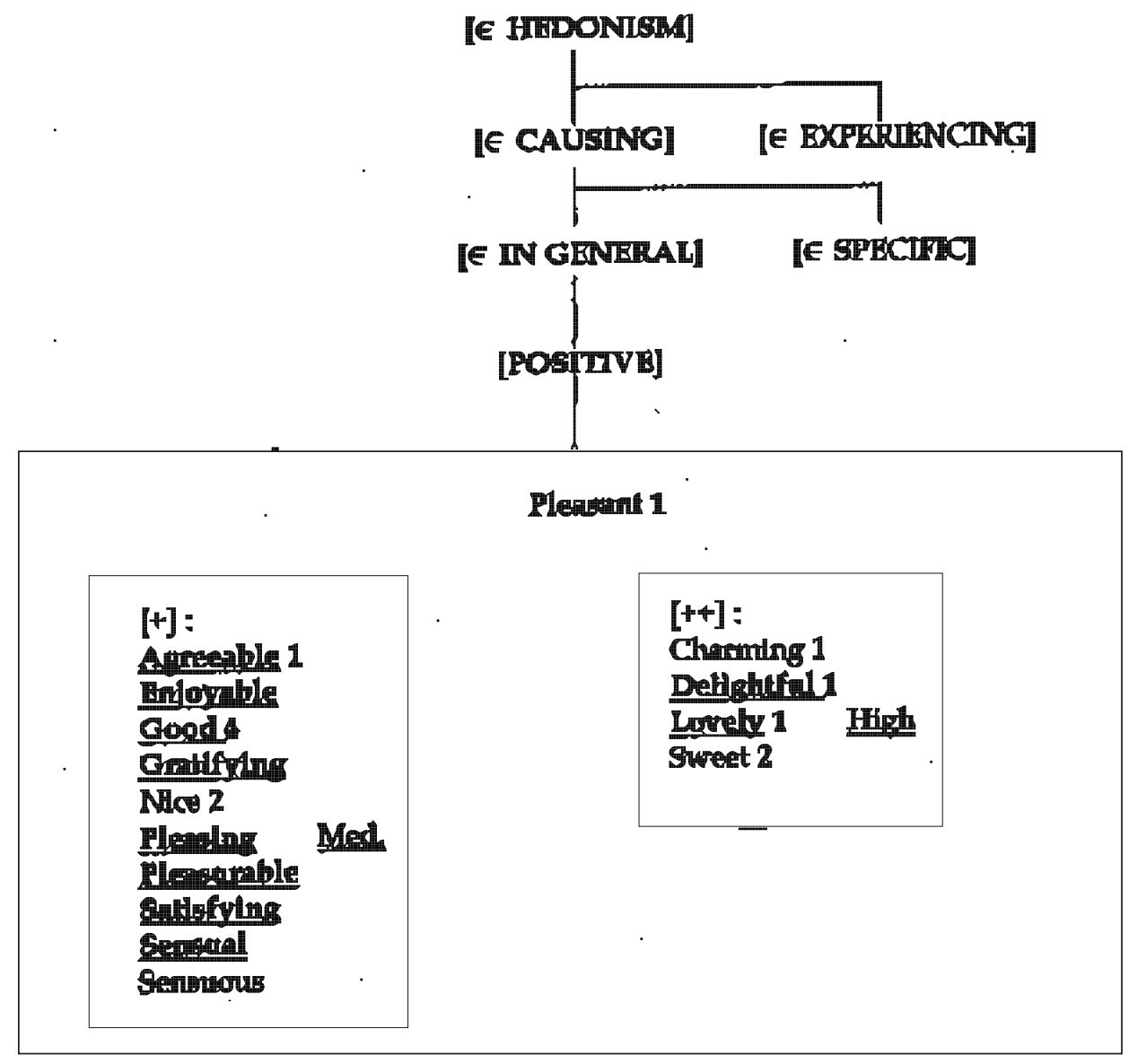


8.2. La superdimensión emotivo-conductual combinada:

[ $\in$ EMOTION/BEHAVIOUR COMBINED]

\subsubsection{La dimensión del afecto: $[\in$ AFFECTION].}

8.2.3.1. Subdimensión [ $\in$ LIKING]

8.2.3.1.3.- Grupo [ $\in$ RECIPIENT]

8.2.3.2. Subdimensión $[\in$ LOVE]

8.2.3.2.1. Grupo $[\in$ SEXUAL] $[\in$ AGENT]

8.2.3.2.3.3.- Subgrupo [ $\in$ RECIPIENT]

GRAFICO DE LA ESCALA DIMENSIONAL POSITIVA

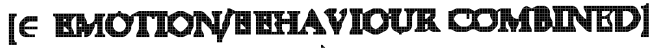

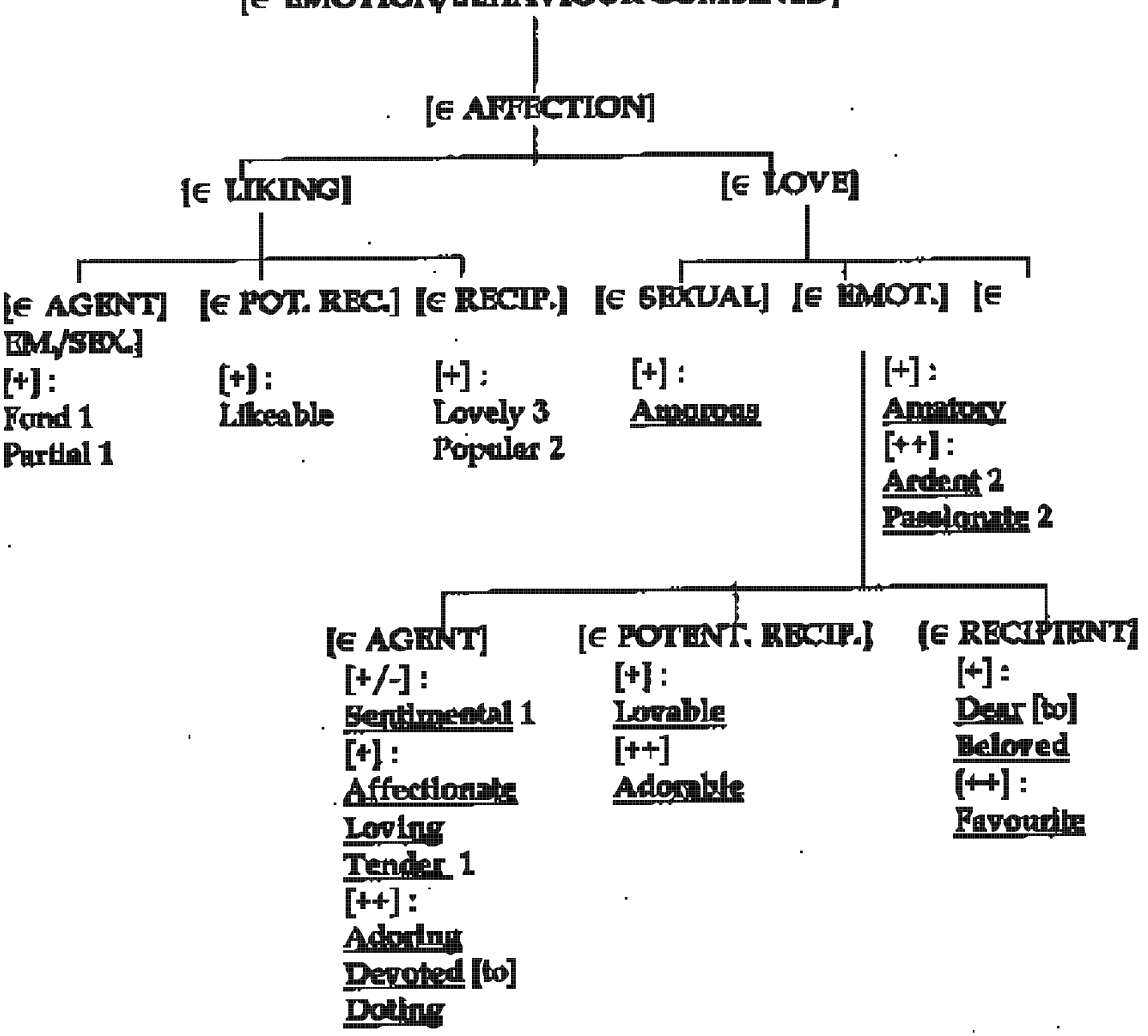

[NB: creemos que emotional debería ser también incluido en el grupo [ $\in$ EMOT.], subgr. [ $\in$ AGENT] de la subdimensión del amor] 
10.4. La dimensión del deseo,

10.4.1. La subdimensión [ $\in$ FEELING DESIRE].

10.4.1.1. Grupo [ $\in$ FEELING DESIRE IN GENERAL].

10.4.1.4. Grupo $[\in$ FEELING DESIRE FOR SEX].

10.4.1.5. Representación gráfica de la subdimensión.

\section{GRAFICO DE LA SUBDIMENSION}

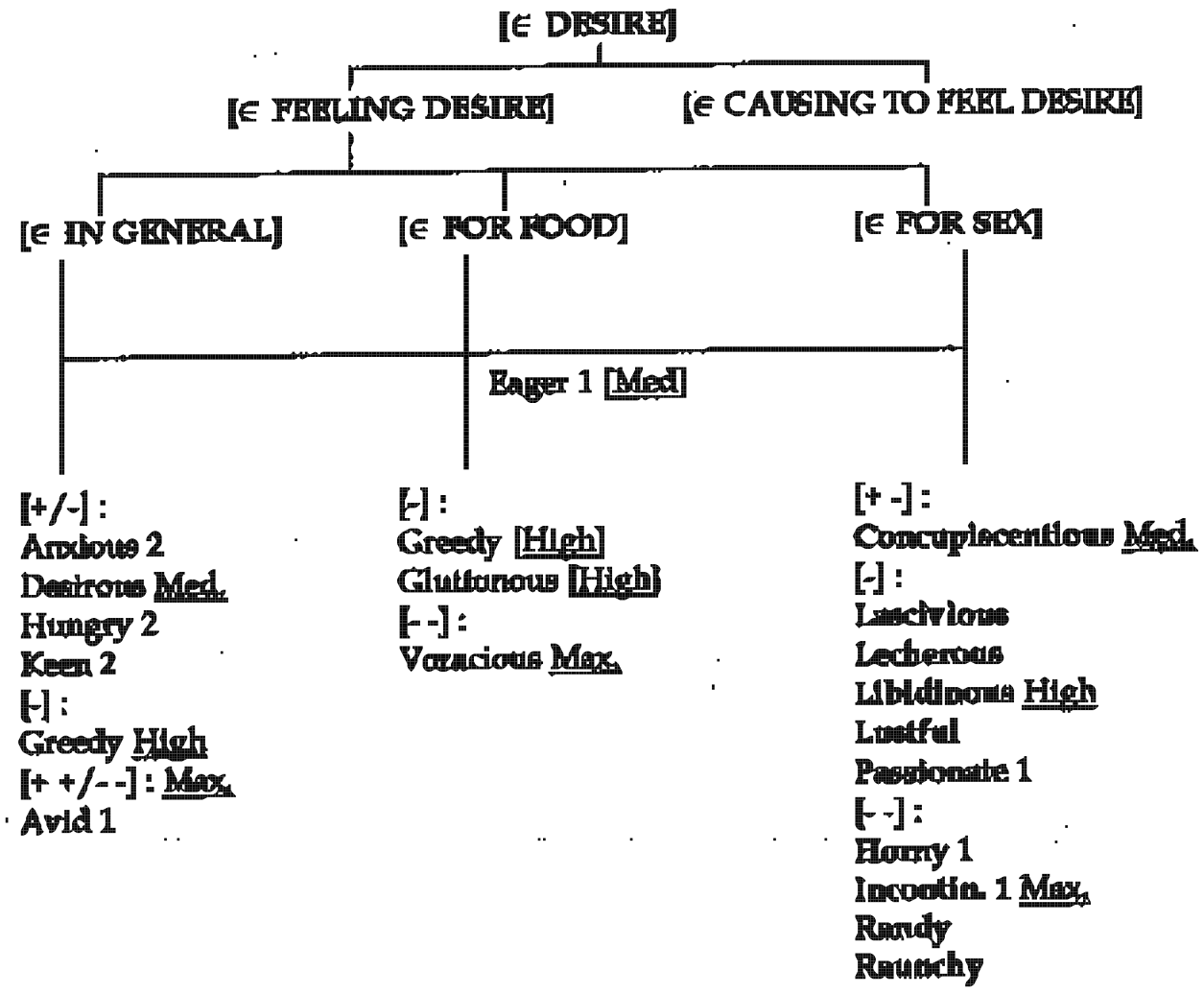

NB.Creemos que se podría añadir también sexual en [ $\in$ FEELING DESIRE FOR SEX] por su relación con sex como emoción erótica (cfr. definiciones de la estructuración) 
10.4.2. La subdimensión [ $\in$ CAUSING TO FEEL DESIRE]

10.4.2.1. El grupo [ $\in$ CAUSING TO FEEL DESIRE FOR SEX].

GRAFICO ARBOREO DE LA SUBDIMENSION:

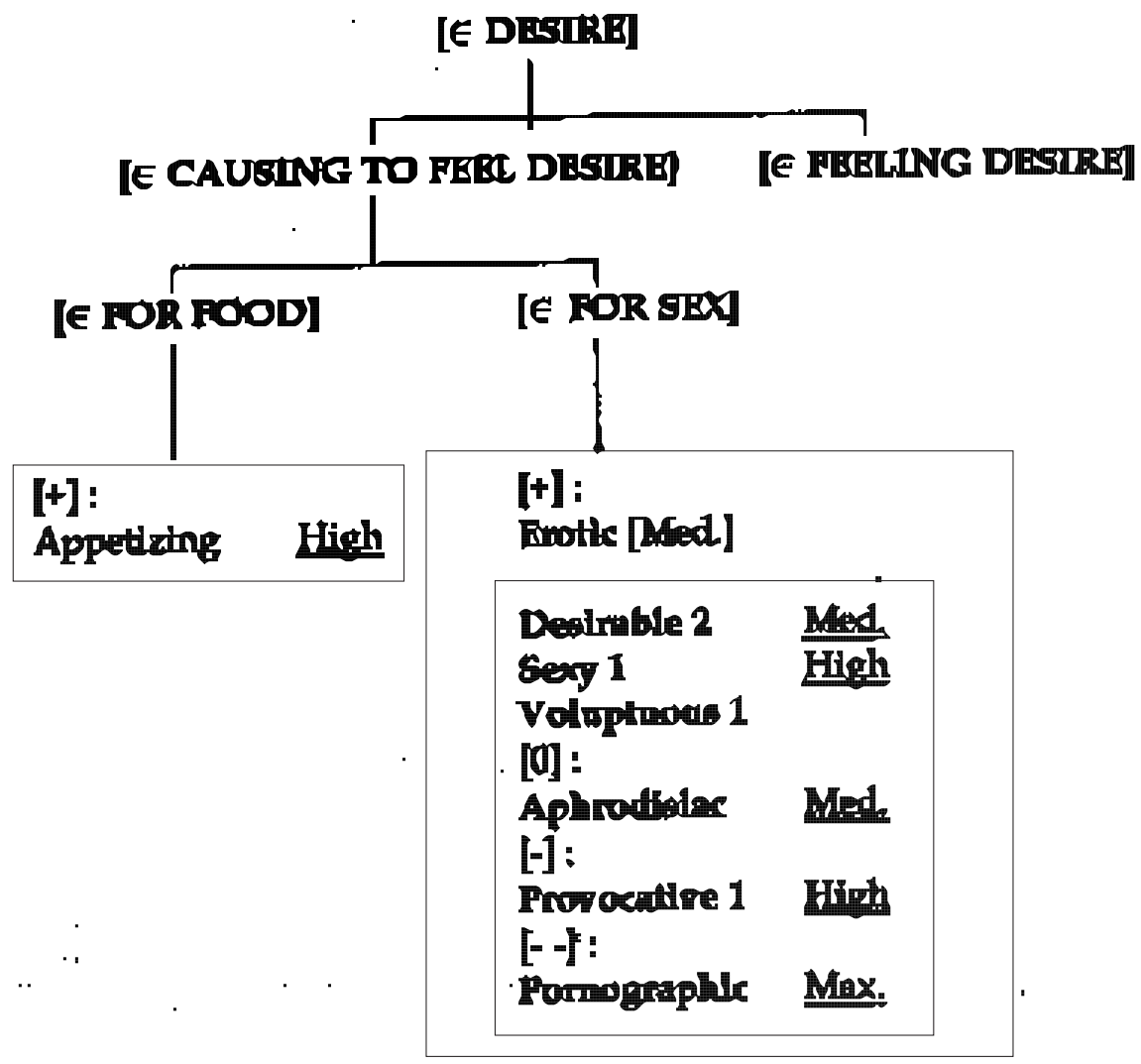


GRAFICO DE TODA LA DIMENSION:

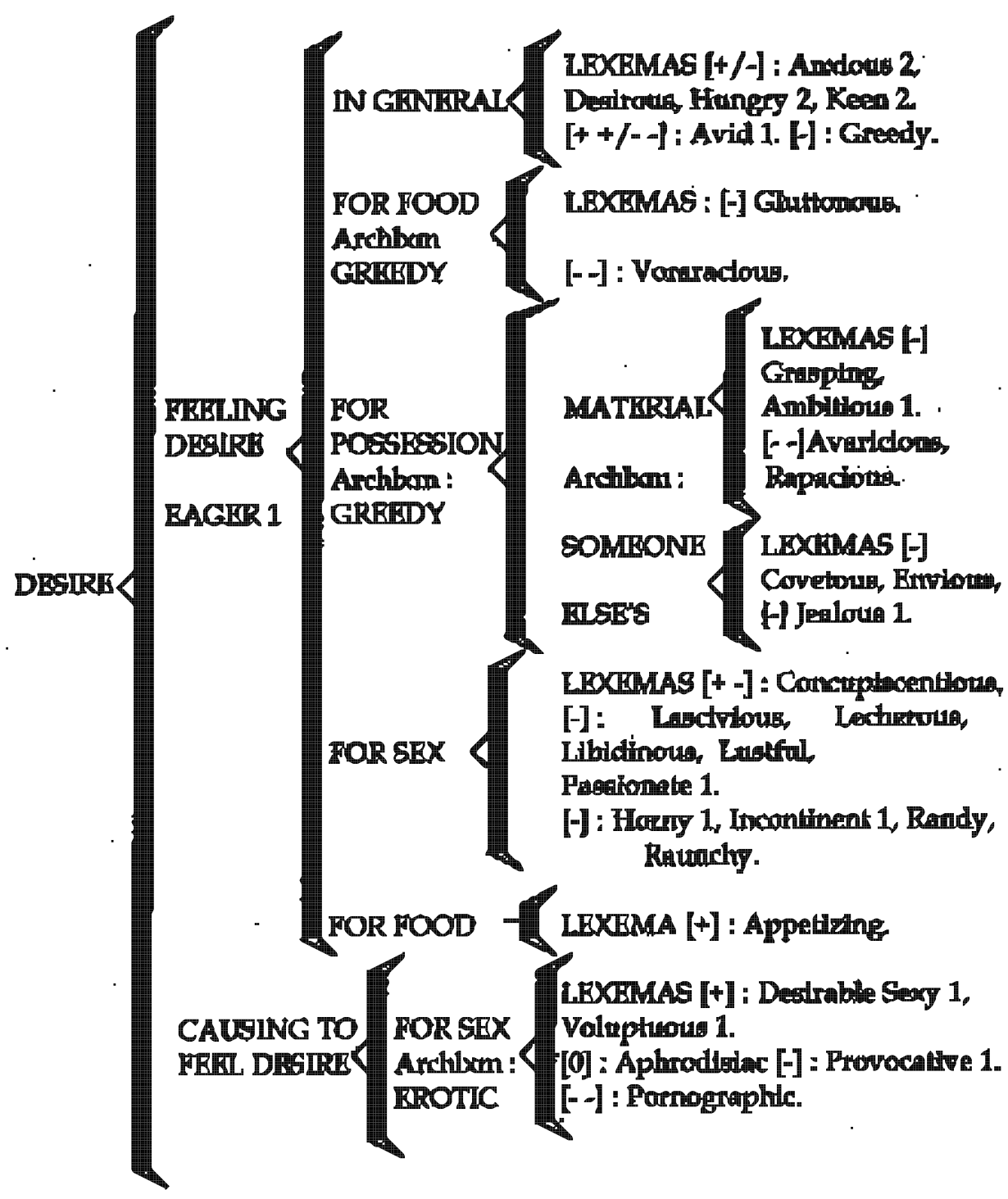

